\title{
Relações cálcio:magnésio e características químicas do solo sob cultivo de soja e milho
}

\author{
Anderson LANGE ${ }^{1 *}$, Edilson CAVALLI², Cassiano Spaziani PEREIRA, \\ Marcos Vinicius CHAPLA ${ }^{1}$, Onã da Silva FREDDI ${ }^{1}$ \\ ${ }^{1}$ Instituto de Ciências Agrárias e Ambientais, Universidade Federal de Mato Grosso, Sinop, MT, Brasil. \\ ${ }^{2}$ Centro de Solos e Recursos Ambientais, Instituto Agronômico, Campinas, SP, Brasil. \\ *E-mail: paranalange@hotmail.com \\ (Orcid: 0000-0002-2518-1915; 0000-0003-0824-0861; 0000-0002-2001-697X; \\ 0000-0002-9731-7586; 0000-0002-1617-6954)
}

Recebido em 05/01/2021; Aceito em 09/07/2021; Publicado em 14/07/2021.

\begin{abstract}
RESUMO: Estudou-se as relações Ca:Mg no solo e o desenvolvimento da soja e milho. Conduziu-se em vasos com solo seis tratamentos variando a relação Ca:Mg no solo de 01:01, 02:01, 03:01, 04:01, 05:01 e 06:01 e cinco repetições, por meio da incubação do solo com calcário calcítico e dolomítico, na dose final de 8 ton ha ${ }^{-1}$. O solo foi incubado e amostrado aos 45, 90, 200 e 300 dias. Após os 90 dias de incubação semeou-se a soja a qual foi colhida no final do ciclo. Em sequência semeou-se o milho, o qual também foi colhido. As relações Ca: $\mathrm{Mg}$ mais altas no solo ( $\geq 04: 01)$ durante o cultivo da soja afetaram positivamente a altura e a massa das plantas; já a produção final de grãos foi superior nas relações 02:01, 04:01 e 01:01, resultado da quantidade de vagens e da massa de mil grãos. No milho a massa da planta e a quantidade de grãos por espiga foram superiores nas relações $\geq 04: 01$ e a produção final foi inferior na relação 06:01. Conclui-se que a soja pode ser cultivada numa relação Ca:Mg mais ampla e no milho, relações entre 03:01 a 05:01, favorecem a estrutura vegetativa da planta e produção.
\end{abstract}

Palavras-chave: calagem; incubação; nutrição; calcário.

\section{Calcium:magnesium ratio and chemical characteristics of soil under crop of soy and corn}

\begin{abstract}
Soil Ca:Mg ratios and soybean and corn development were studied. Six treatments were conducted in pots with soil, varying the Ca:Mg ratio in the soil from 01:01, 02:01, 03:01, 04:01, 05:01 and 06:01 and five repetitions, by incubating the soil with calcitic and dolomitic limestone, in the final rate of 8 ton ha ${ }^{-1}$. The soil was incubated and sampled at 45, 90, 200 and 300 days. After 90 days of incubation, soy was sown, which was harvested at the end of the cycle. In sequence, corn was sown, which was also harvested. The highest Ca: $\mathrm{Mg}$ ratios in the soil $(\geq 04: 01)$ during soybean cultivation positively affected plant height and mass; the final grain production was higher in the 02:01, 04:01 and 01:01 ratios, the result of the quantity of pods and the mass of a thousand grains. In corn, the plant mass and the amount of grains per ear were higher in the ratio $\geq 04: 01$ and the final production was lower in the ratio 06:01. It is concluded that soybeans can be grown in a wider Ca: $\mathrm{Mg}$ ratio and in corn, ratios between 03:01 to 05:01, favor the vegetative structure of the plant and production.
\end{abstract}

Keywords: liming; incubation; nutrition; limestone.

\section{INTRODUÇÃO}

O desenvolvimento e desempenho produtivo das culturas agrícolas em solos ácidos e com baixa saturação de bases é limitado pela da acidez e da deficiência dos nutrientes cálcio (Ca) e magnésio (Mg), havendo a necessidade da adição de corretivos nestas condições. Assim, os corretivos podem ser escolhidos não apenas por seu poder de neutralização da acidez, mas também pela proporção entre cátions acompanhantes, principalmente a relação entre $\mathrm{Ca}$ e $\mathrm{Mg}$ no material.

O Ca é imprescindível para manter a integridade estrutural e funcional das membranas, das paredes celulares e, quando há deficiência do mesmo, as membranas permitem o efluxo do conteúdo citoplasmático, ocorrendo redução de crescimento de tecidos meristemáticos, prejuízos nas extremidades de crescimento e folhas novas, que se tornam deformadas e cloróticas Malavolta et al. (1997). Na soja, a deficiência de Ca afeta os pontos de crescimento, atrofiando o sistema radicular e matando a gema apical, retardando a emergência das primeiras folhas, e quando emergem apresentam encarquilhamento, além do colapso do pecíolo (SEDIYAMA, 2009).

O Mg apresenta participação estrutural na clorofila e ativador enzimático em muitos processos da vida das plantas e co-fator de quase todas as enzimas fosforilativas que forma uma ponte entre o ATP e o ADP e a molécula da enzima. A transferência de energia desses dois compostos é fundamental nos processos da fotossíntese, respiração, reações de síntese de compostos orgânicos, absorção iônica 
e trabalho mecânico, além do aprofundamento e expansão da raiz (MALAVOLTA et al., 1997).

A quantidade de calcário a ser aplicada dependerá da análise do solo, tamanho da partícula do calcário, teor de $\mathrm{MgO}$ e $\mathrm{CaO}$, textura do solo, requisitos da cultura e sistema de produção e do método usado na recomendação da calagem (TOME JUNIOR, 1996). O suprimento de Ca e Mg está normalmente vinculado à aplicação de calcário calcítico que contêm, em média, $45 \%$ de $\mathrm{CaO}$ e menos que $5 \%$ de $\mathrm{MgO}$, e de calcário dolomítico, que contém em média, 20 a $40 \%$ de $\mathrm{CaO}$ e 10 a 20\% de $\mathrm{MgO}$. Conforme Moore et al. (1961), o excesso de Ca em relação ao Mg na solução do solo prejudica a absorção deste último, assim como o excesso de $\mathrm{Mg}$ também prejudica a absorção de Ca.

A relação e a proximidade entre os nutrientes $\mathrm{Ca}$ e $\mathrm{Mg}$ na nutrição de plantas estão ligadas às suas propriedades químicas próximas (raio iônico, valência, grau de hidratação e mobilidade), fazendo com que haja competição pelos sítios de adsorção no solo e na absorção pelas raízes. Como consequência, a presença de um pode prejudicar os processos de adsorção e absorção do outro e apresentando o Ca maior preferência em relação ao $\mathrm{Mg}$ no complexo de troca do solo (YADARE; GIRDHAR, 1981).

Em relação a exigência de $\mathrm{Ca}$ e $\mathrm{Mg}$ e seus teores em tecidos no milho, Duarte et al. (2018), publicaram níveis de referência de nutrientes nos grãos diferentes dos apresentados em boletins mais antigos, o que pode alterar a exigência para alguns elementos na cultura.

Gallo et al. (1956), em trabalho pioneiro no solo inicialmente com 3,63 e 0,87 $\mathrm{cmol}_{\mathrm{c}} \mathrm{dm}^{-3}$ de $\mathrm{Ca}$ e $\mathrm{Mg}$, respectivamente, testaram a dose de calcário para elevar a saturação por bases à 70\% ou o dobro da dose e verificaram que a soja se desenvolveu melhor ao usar calcário dolomítico versus calcítico, e que ao dobrar a dose, o desenvolvimento foi ainda melhor. Holzschuh (2007) não verificou diferença para produtividade da soja em três localidades ao variar as relações Ca: $\mathrm{Mg}$ tanto com calagem superficial ou incorporada. Moreira et al. (1999), cultivaram cevada sob altas quantidades de $\mathrm{Ca}$ e $\mathrm{Mg}$ no solo verificaram inibição competitiva na absorção de potássio por $\mathrm{Ca}$ e $\mathrm{Mg}$.

Em milho, os resultados são variados. Silva (1980) verificou em dois solos distintos maior produção para o quando a relação Ca:Mg era próxima a 03:01 comparada a 01:01. Clark et al. (1997) verificaram redução nas concentrações de $\mathrm{Ca}, \mathrm{P}$ e $\mathrm{Mn}$ da parte aérea, com o aumento da aplicação de $\mathrm{Mg}$, enquanto para potássio $(\mathrm{K})$ não houve diferença e, que os maiores acúmulos de massa radicular e aérea ocorreram para relações superiores a 10:01 de Ca:Mg. Munoz Hernandez; Silveira (1998) verificaram que quando o solo apresentava relação Ca: $\mathrm{Mg}$ maior 03:01, havia redução no crescimento e queda na produção da gramínea decorrente do antagonismo que o Ca causa na absorção de $\mathrm{Mg}$ e Medeiros et al. (2008) ao estudarem relações de até 32:01 concluíram que a altas concentração de $\mathrm{Ca}$ reduzem a absorção de $\mathrm{Mg}$ e K e também a produção.

Com base no que foi descrito, realizou-se o presente estudo com o propósito de avaliar a influência de relações $\mathrm{Ca}: \mathrm{Mg}$ no solo no desenvolvimento e produção da soja e do milho e nas características químicas do solo.

\section{MATERIAL E MÉTODOS}

O experimento foi instalado em casa de vegetação e o solo utilizado foi classificado como Latossolo Vermelho Amarelo
Distrófico (LvAd), coletado em área de mata, na região de Sinop - MT, com as seguintes características químicas antes da instalação: $\mathrm{pH}=4,37 ; \mathrm{P}=0,63 \mathrm{mg} \mathrm{dm}^{-3}, \mathrm{~K}=32,0 \mathrm{mg} \mathrm{dm}^{-3}$; Ca $=0,31 \mathrm{cmol}_{\mathrm{c}} \mathrm{dm}^{-3} ; \mathrm{Mg}=0,27 \mathrm{cmol}_{\mathrm{c}} \mathrm{dm}^{-3} ; \mathrm{Al}=0,7 \mathrm{cmol}_{\mathrm{c}} \mathrm{dm}^{-}$ 3; $\mathrm{H}+\mathrm{Al}=9,18 \mathrm{cmol}_{\mathrm{c}} \mathrm{dm}^{-3} ; \mathrm{CTC}=9,83 \mathrm{cmol}_{\mathrm{c}} \mathrm{dm}^{-3} ; \mathrm{V}=$ $6,69 \%$.

Foi utilizado o delineamento inteiramente casualizado (DIC), com 6 tratamentos e 5 repetições, variando doses de calcário calcítico e dolomítico, para estabelecer crescentes relações finais de $\mathrm{Ca}: \mathrm{Mg}$ no solo, a saber: 01:01, 02:01, 03:01, 04:01, 05:01 e 06:01, totalizando 30 vasos de $15 \mathrm{dm}^{-3}$ de volume cada. A dose de calcário (PRNT médio de 74\%) foi fixada em 8 ton $\mathrm{ha}^{-1}$, proporcional ao volume de solo do vaso, o que teria potencial de elevar a saturação por bases até $67 \%$, conforme o método da saturação por bases (Raij, 1996). O cálculo da quantidade de cada calcário foi definido estequiometricamente, misturando calcário calcítico PRNT $=74 \%(46 \%$ de $\mathrm{CaO}$ e $3 \%$ de $\mathrm{MgO}$ ) e dolomítico (29\% de $\mathrm{CaO}$ e $19 \%$ de $\mathrm{MgO}$ ), PRNT $=74,5 \%$ (Tabela 1$)$.

Tabela 1. Quantidades de calcário aplicadas, valores teóricos de cálcio e magnésio após a reação do calcário e a relação $\mathrm{Ca}: \mathrm{Mg}$ final teórica. Table 1. Quantities of lime applied, theoretical values of calcium and magnesium after the lime reaction and the theoretical final $\mathrm{Ca}: \mathrm{Mg}$ ratio.

\begin{tabular}{|c|c|c|c|c|c|}
\hline \multirow{2}{*}{$\begin{array}{l}\text { TRAT } \\
\text { (Ca:Mg) }\end{array}$} & Calcítico & Dolomítico & $\mathrm{Ca}$ & $\mathrm{Mg}$ & \multirow{2}{*}{ Rel.final Ca:Mg } \\
\hline & \multicolumn{2}{|c|}{ ton $\mathrm{ha}^{-1}$} & \multicolumn{2}{|c|}{ cmolc dm ${ }^{-3}$} & \\
\hline $01: 01$ & 0,00 & 8,00 & 4,46 & 4,04 & 1,1 \\
\hline 02:01 & 3,40 & 4,60 & 5,50 & 2,69 & 2,0 \\
\hline 03:01 & 5,10 & 2,90 & 6,01 & 2,02 & 3,0 \\
\hline 04:01 & 6,20 & 1,80 & 6,35 & 1,58 & 4,0 \\
\hline 05:01 & 6,85 & 1,20 & 6,55 & 1,32 & 5,0 \\
\hline 06:01 & 7,35 & 0,65 & 6,70 & 1,12 & 6,0 \\
\hline
\end{tabular}

O propósito deste estudo se deve as condições químicas em que os solos mato-grossenses se encontram em relação aos nutrientes cálcio e magnésio e as culturas escolhidas - soja e milho - pela expressão da área cultivada. Um levantamento foi realizado com aproximadamente 250 mil resultados de análises de solo catalogadas entre os anos de 2017 e 2019 em solos mato-grossenses (Tabela 2), apontando que cerca de $59 \%$ delas apresentavam teores de $\mathrm{Ca} \leq 2,40 \mathrm{cmol}_{\mathrm{c}} \mathrm{dm}^{-3}$, $65 \%$ com teores de $\mathrm{Mg} \leq 0,90 \mathrm{cmol}_{\mathrm{c}} \mathrm{dm}^{-3}$ e em $57 \%$ a relação $\mathrm{Ca}: \mathrm{Mg} \leq$ 03:01, resultado do consumo ano a ano de calcário dolomítico nas propriedades, correspondendo a 95\% das vendas do insumo.

Tabela 2. Distribuição de teores de cálcio (Ca) e magnésio (Mg), relação Ca:Mg e percentual em relação ao total de 250 mil amostras de terra colhidas no Estado (resultados cedidos por três laboratórios estaduais).

Table 2. Distribution of calcium (Ca) and magnesium $(\mathrm{Mg})$ contents, $\mathrm{Ca}: \mathrm{Mg}$ ratio and percentage in relation to the total of 250 thousand soil samples collected in the State (results provided by three state laboratories)

\begin{tabular}{cccccc}
\hline${ }^{1} \mathrm{Ca}\left(\mathrm{cmolc} \mathrm{dm}^{-3}\right)$ & $\%$ & ${ }^{1} \mathrm{Mg}\left(\mathrm{cmolc} \mathrm{dm}^{-3}\right)$ & $\%$ & $\mathrm{Ca}: \mathrm{Mg}$ & $\%$ \\
\hline$<0,40$ & 2 & $<0,15$ & 2 & $<1,5 / 1$ & 4 \\
0,40 a 1,20 & 11 & 0,16 a 0,45 & 18 & $1,5-2,0 / 1$ & 11 \\
1,21 a 2,40 & 45 & 0,46 a 0,90 & 45 & $2,0-3,0 / 1$ & 42 \\
2,41 a 4,0 & 37 & 0,91 a 1,5 & 29 & $3,0-4,0 / 1$ & 24 \\
$>4,0$ & 5 & $>1,5$ & 6 & $>4,0$ & 19 \\
\hline & ${ }^{1}$ distribuição dos teores proposta por Alvarez et al. (1999)
\end{tabular}

O calcário foi adicionado ao solo no dia 15/04/2015, o qual foi irrigado semanalmente. Aos 45 e 90 dias o solo dos 
vasos foi amostrado, retirando-se quatro furos por vaso para compor uma amostra (repetição), aos 45 dias procedeu-se a análise apenas para cálcio e magnésio e aos 90 dias a análise básica completa ( $\mathrm{pH}$ em água, $\mathrm{P}, \mathrm{K}, \mathrm{Ca}, \mathrm{Mg}, \mathrm{H}+\mathrm{Al}$ ).

Em 18/10/2015 o solo voltou a ser irrigado e em 21/10 foi semeada a soja, cultivar TMG 132, de ciclo normal, com cinco sementes por vaso. Em V3 realizou-se o desbaste, deixando apenas três plantas por vaso. Antes da semeadura, realizou-se a adubação de base por vaso, quando os fertilizantes foram distribuídos por vaso e homogeneizado em todo o volume. A adubação foi feita utilizando uma formulação comercial MAP ( $51 \%$ de $\mathrm{P}_{2} \mathrm{O}_{5}$ e $9 \%$ de $\mathrm{N}$ ), visando atingir a concentração de $250 \mathrm{mg} \mathrm{dm}^{-3}$ de P. A aplicação dos micronutrientes foi com diluição em água e posterior distribuição no solo nas concentrações de; Boro 0,5 $\mathrm{mg} \mathrm{dm}^{-3}$, Manganês $4 \mathrm{mg} \mathrm{dm}^{-3}$, Cobre $1,5 \mathrm{mg} \mathrm{dm}^{-3}$ e Zinco $5 \mathrm{mg} \mathrm{dm}^{-3}$. A adubação de cobertura foi feita utilizando a mesma metodologia dos micronutrientes, com cloreto de potássio e a ureia, parcelados em 3 aplicações até aos 45 dias, visando atingir de $250 \mathrm{mg} \mathrm{dm}$ 3 de potássio e nitrogênio finais, respectivamente (MALAVOLTA, 1980).

O tratamento das sementes de soja foi realizado com a aplicação, inoculante, cobalto, molibdênio e inseticida a base de fipronil. O controle de pragas e doenças foi realizado conforme a necessidade da cultura.

Em 10/02/2016, a soja foi colhida e avaliou-se: altura de plantas, diâmetro do caule, altura de inserção da $1^{\mathrm{a}}$ vagem, massa seca da parte aérea, massa seca de raiz, número de vagens por planta, número de grãos por planta, vagens com um, dois ou três grãos, relação parte aérea:raiz, massa de 1000 grãos (estimada com subamostras de 50 grãos cada), produção de grãos e concentração e acúmulo de Ca e $\mathrm{Mg}$ na planta. O solo foi amostrado seguindo mesmo procedimento anterior, contabilizando nesta data $\sim 200$ dias do início da incubação. Após a amostragem os vasos foram mantidos sem irrigação por um ano.

Em fevereiro de 2017, o solo foi fertilizado com fósforo e novamente irrigado e o milho DKB 290 foi semeado, com quatro sementes por vaso e desbaste após 15 dias, conduzindo duas plantas durante a avaliação. As sementes receberam tratamento industrial e o controle de pragas e doenças foi realizado conforme a necessidade da cultura.

A fertilização para o milho foi similar a soja, apenas com aumento da dose de $\mathrm{N}$ para $300 \mathrm{mg} \mathrm{dm}^{-3}$. Para o milho avaliouse: a altura das plantas, número de folhas, diâmetro do colmo, diâmetro da espiga, grãos por fileiras, grãos por espiga, massa seca da raiz, massa da parte aérea, relação parte aérea:raiz, massa de 1000 grãos (estimada com subamostras de 50 grãos cada) e a produção de grãos. Após a colheita do milho o solo foi amostrado e analisado, finalizando o estudo com aproximadamente 300 dias de incubação de solo úmido, descontando o período de repouso dos vasos (sem irrigação).

Os dados obtidos foram analisados estatisticamente no programa estatístico SISVAR ${ }^{\circledR}$ (FERREIRA, 2011), sendo submetido a análise de variância pelo teste $\mathrm{F}$ a $5 \%$ de probabilidade. As características que apresentaram diferenças significativas foram submetidas ao teste Scott Knott 5\% de probabilidade.

\section{RESULTADOS}

\subsection{Atributos Químicos do Solo}

Serão apresentados apenas resultados significativos para as variáveis referentes ao solo, a exceção do valor de saturação por bases (V\%), que em alguma situação, apesar de não significativo, contribui para a discussão.

Observa-se na Tabela 3, que houve efeito significativo dos tratamentos já na primeira avaliação aos 45 dias de incubação e verificou-se que os valores de Ca e $\mathrm{Mg}$ passaram de 0,31 e 0,27, valores iniciais do solo de mata, para no máximo 4,35 e 2,96 $\mathrm{cmol}_{\mathrm{c}} \mathrm{dm}^{-3}$, não atingindo os valores teóricos propostos máximos de 6,7 e $4,04 \mathrm{cmol}_{\mathrm{c}} \mathrm{dm}^{-3}$, respectivamente. Já as relações Ca:Mg buscadas ficaram muito próximas as pretendidas já na primeira avaliação.

Tabela 3. Valores médios de cálcio (Ca), magnésio (Mg), relação Ca:Mg, pHágua, saturação por bases (V\%), \% de cálcio na CTC (CaCTC) e \% de $\mathrm{Mg}$ na $\mathrm{CTC}\left(\mathrm{Mg}_{\mathrm{CTC}}\right)$ aos 45 e 90 dias após a aplicação de calcário para calibrar as relações Ca:Mg no solo.

Table 3. Average values of calcium (Ca), magnesium (Mg), Ca:Mg ratio, $\mathrm{pH}_{\text {water, }}$ base saturation (V\%), \% calcium in CEC (CaCTC) and \% $\mathrm{Mg}$ in CEC (Mg CTC) at 45 and 90 days after the application of lime to calibrate the Ca:Mg ratios in the soil.

\begin{tabular}{|c|c|c|c|c|c|c|c|c|c|c|}
\hline \multirow{2}{*}{ TRAT (Ca:Mg) } & $\mathrm{Ca}$ & $\mathrm{Mg}$ & $\mathrm{Ca}: \mathrm{Mg}$ & $\mathrm{pH}$ & $\mathrm{Ca}$ & $\mathrm{Mg}$ & $\mathrm{Ca}: \mathrm{Mg}$ & $\mathrm{V}$ & $\mathrm{Ca}_{\mathrm{CTC}}$ & $\mathrm{Mg}_{\mathrm{CTC}}$ \\
\hline & \multicolumn{3}{|c|}{-- cmolc $\mathrm{dm}^{-3}--$} & \multicolumn{4}{|c|}{-- cmolc dm ${ }^{-3}--$} & \multicolumn{3}{|c|}{ 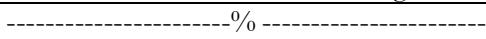 } \\
\hline 01:01 & $2,38 \mathrm{c}$ & $2,96 \mathrm{a}$ & $0,81 \mathrm{c}$ & $5,63 \mathrm{a}$ & $2,79 \mathrm{~d}$ & $2,80 \mathrm{a}$ & $1,09 \mathrm{f}$ & 47,1 & $23,71 \mathrm{~d}$ & $22,87 \mathrm{a}$ \\
\hline 02:01 & $3,42 \mathrm{~b}$ & $1,61 \mathrm{~b}$ & $2,15 \mathrm{~b}$ & $5,47 \mathrm{a}$ & $3,74 \mathrm{c}$ & $1,65 \mathrm{~b}$ & $2,30 \mathrm{e}$ & 45,7 & $31,26 \mathrm{c}$ & $13,97 \mathrm{~b}$ \\
\hline 03:01 & $4,18 \mathrm{a}$ & $1,32 \mathrm{~b}$ & $3,17 \mathrm{~b}$ & $5,34 \mathrm{~b}$ & $4,27 \mathrm{~b}$ & $1,34 \mathrm{~b}$ & $3,19 \mathrm{~d}$ & 49,2 & $37,04 \mathrm{~b}$ & $11,58 \mathrm{~b}$ \\
\hline 04:01 & $4,19 \mathrm{a}$ & $1,01 \mathrm{c}$ & $4,52 \mathrm{a}$ & $5,14 \mathrm{c}$ & $3,92 \mathrm{c}$ & $1,02 \mathrm{c}$ & $3,95 \mathrm{c}$ & 45,9 & $35,96 \mathrm{~b}$ & $9,33 \mathrm{c}$ \\
\hline 05:01 & $4,23 \mathrm{a}$ & $0,85 \mathrm{c}$ & $5,01 \mathrm{a}$ & $5,36 \mathrm{~b}$ & $5,15 \mathrm{a}$ & $1,03 \mathrm{c}$ & $5,08 \mathrm{~b}$ & 56,1 & $46,33 a$ & $9,27 \mathrm{c}$ \\
\hline 06:01 & $4,35 \mathrm{a}$ & $0,86 \mathrm{c}$ & $5,17 \mathrm{a}$ & $5,52 \mathrm{a}$ & $4,57 \mathrm{~b}$ & $0,70 \mathrm{c}$ & $6,53 \mathrm{a}$ & 48,8 & $41,79 \mathrm{a}$ & $6,44 \mathrm{c}$ \\
\hline $\mathrm{CV}$ & 9,29 & 19,0 & 24,2 & 2,0 & 11,5 & 25,6 & 15,4 & 13,6 & 13,7 & 24,5 \\
\hline
\end{tabular}

Médias seguidas da mesma letra na coluna não diferem estatisticamente entre si a $5 \%$ pelo teste de Scott Knott.

Na segunda avaliação, aos 90 dias após a aplicação do calcário, houve efeito para $\mathrm{pH}$, teores de $\mathrm{Ca}, \mathrm{Mg}$, relação Ca:Mg e para as \% das bases Ca e Mg na CTC do solo. O pH variou de 5,1 a 5,6, e os maiores valores foram nas relações $\mathrm{Ca}: \mathrm{Mg}$ de 01:01 e 06:01. Os teores de Ca e Mg seguiram a tendência proposta nos tratamentos, com maiores valores de Ca no tratamento 06:01 (4,57 $\left.\mathrm{cmol}_{\mathrm{c}} \mathrm{dm}^{-3}\right)$ com a maior quantidade de calcário calcítico, e o maior valor de $\mathrm{Mg}$ na para a maior dose de dolomítico, no tratamento 01:01 (2,80 $\left.\mathrm{cmol}_{\mathrm{c}} \mathrm{dm}^{-3}\right)$, ambas aquém do valor teórico calculado. $\mathrm{O}$ mesmo aconteceu com a saturação por bases no solo (V\%), em que em média ficou próximo a 49\%, sendo o almejado de $67 \%$, quando do cálculo da aplicação de 8 toneladas por hectare, com os calcários acima citados. As relações $\mathrm{Ca}: \mathrm{Mg}$ sofreram grande alteração em relação ao solo inicial, reagindo conforme o proposto, variando de 1,09 até 6,53 (Ca:Mg). Decorrente destas variações, as \% de Ca e de $\mathrm{Mg}$ na CTC também seguem a mesma tendência, tendo a \% de Ca variado 
de 41,8 a 23,7, sendo significativamente maior para a maior relação Ca: $\mathrm{Mg}$ pretendida (tratamento 06:01), o que também aconteceu com a \% de $\mathrm{Mg}$, com valor significativamente maior no tratamento 01:01, com $\sim 23 \%$ de Mg na CTC.

$\mathrm{Na}$ terceira e quarta avaliações, aos 200 e 300 dias de incubação (Tabela 4), houve efeito novamente para $\mathrm{pH}$, teores de $\mathrm{Ca}, \mathrm{Mg}$, relação $\mathrm{Ca}: \mathrm{Mg}$ e nas \% Ca e $\mathrm{Mg}$ na CTC do solo, sendo que para o $\mathrm{V} \%$ o efeito significativo foi apenas aos 200 dias, mesmo assim agronomicamente e numericamente os valores ficaram muito próximos não variando mais que 2 unidades. $\mathrm{O}$ pH do solo aos 200 dias variou de 5,93, no tratamento 01:01 até 5,44 no tratamento 04:01, não havendo relação direta com os tratamentos. Aos 300 dias também houve diferença no $\mathrm{pH}$, porém já com maior uniformidade nos valores e maior acidificação decorrente da aplicação de $\mathrm{N}$ no milho e da extração de nutrientes, ficando a média próximo a 4,25. Os teores de Ca e $\mathrm{Mg}$ aos 200 e 300 dias seguiram a tendência das aplicações de calcário, com maiores valores de Ca no tratamento 06:01 (3,71 e 4,04 $\left.\mathrm{cmol}_{\mathrm{c}} \mathrm{dm}^{-3}\right)$ e o maior valor de $\mathrm{Mg}$ no tratamento 01:01 (2,40 e 3,01 $\left.\mathrm{cmol}_{\mathrm{c}} \mathrm{dm}^{-3}\right)$, respetivamente para as duas avaliações, sem se aproximar do valor teórico calculado. O V\% do solo aos 200 ficou próximo a 49,2\%, sendo significativamente superior no tratamento 01:01 e aos 300 dias atingiu na média dos vasos, $44,4 \%$, sem diferença entre os tratamentos.

As relações Ca: $\mathrm{Mg}$ aos 200 dias tiveram comportamento similar a amostragem de 90 dias, já aos 300 dias as relações começaram a se estreitar, com a entrada de mais magnésio no sistema em relação a cálcio.

Tabela 4. Valores médios de $\mathrm{pH}_{\text {água }}$, cálcio (Ca), magnésio (Mg), relação Ca:Mg, saturação por bases (V\%), \% de cálcio na CTC (CaCTC) e \% de Mg na CTC (MgстC) aos 200 e 300 dias após a aplicação de calcário para calibrar as relações Ca:Mg no solo.

Table 4. Average values of $\mathrm{pH}_{\text {water, }}$, calcium (Ca), magnesium (Mg), Ca:Mg ratio, base saturation (V\%), \% calcium in CEC (CaCTC) and \% $\mathrm{Mg}$ in CEC (MgCTC) at 200 and 300 days after the application of lime to calibrate the Ca:Mg ratios in the soil.

\begin{tabular}{|c|c|c|c|c|c|c|c|c|c|c|c|c|c|c|}
\hline TRAT & $\mathrm{pH}$ & $\mathrm{Ca}$ & $\mathrm{Mg}$ & Ca:Mg & $\mathrm{V}$ & Састс & $\mathrm{Mg}_{\mathrm{CTC}}$ & $\mathrm{pH}$ & $\mathrm{Ca}$ & $\mathrm{Mg}$ & $\mathrm{Ca}: \mathrm{Mg}$ & $\mathrm{V}$ & Састс & $\mathrm{Mg}_{\mathrm{CTC}}$ \\
\hline (Ca:Mg) & \multicolumn{4}{|c|}{ - cmolc $\mathrm{dm}^{-3}$ - } & \multicolumn{3}{|c|}{------- \% ------ } & \multicolumn{4}{|c|}{ - cmolc $\mathrm{dm}^{-3}$} & \multicolumn{3}{|c|}{ 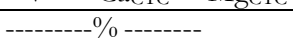 } \\
\hline 01:01 & $5,93 \mathrm{a}$ & $2,26 \mathrm{e}$ & $2,40 \mathrm{a}$ & $094 \mathrm{f}$ & $50,5 \mathrm{a}$ & $24,14 \mathrm{f}$ & $25,59 \mathrm{a}$ & 454 & $2,66 \mathrm{c}$ & $3,01 \mathrm{a}$ & & 46,7 & $21,2 \mathrm{~d}$ & $24,0 \mathrm{a}$ \\
\hline 02:01 & $5,70 \mathrm{c}$ & 2 & 1,80 & & & & & & & & & & & \\
\hline 03:01 & $5,64 \mathrm{c}$ & $27 \mathrm{c}$ & 1,29 & & 48,8 & & & & & & & 4 & & $13,3 \mathrm{c}$ \\
\hline 0 & $5,44 \mathrm{~d}$ & $3,57 \mathrm{~b}$ & 1,03 & & $49,8 \mathrm{a}$ & & & & & & & 43 & & $10,9 \mathrm{~d}$ \\
\hline 0 & $5,65 \mathrm{c}$ & $3,71 \mathrm{a}$ & $0,77 \mathrm{e}$ & & $48,2 \mathrm{~b}$ & 39,3 & & & $3,51 \mathrm{~b}$ & 0,9 & & 41, & & $8,2 \mathrm{e}$ \\
\hline 06:01 & $5,82 \mathrm{~b}$ & $3,71 \mathrm{a}$ & $0,56 \mathrm{f}$ & $9 \mathrm{a}$ & $48,4 \mathrm{~b}$ & 41,3 & $6,29 \mathrm{f}$ & $4,04 \mathrm{~b}$ & $4,10 \mathrm{a}$ & $0,92 \mathrm{~d}$ & & 44 & 35,2 a & $7,9 \mathrm{e}$ \\
\hline $\mathrm{CV}$ & 1,9 & 3,0 & 3,7 & 4,64 & 1,87 & 1,75 & 2,81 & 5,8 & 8,19 & 15,61 & 9,72 & 8,93 & 7,95 & 11,43 \\
\hline
\end{tabular}

Médias seguidas da mesma letra na coluna não diferem estatisticamente entre si a $5 \%$ pelo teste de Scott Knott. Valores médios aos 200 dias de $\mathrm{P}=36 \mathrm{mg}$ $\mathrm{dm}^{-3}, \mathrm{~K}=32 \mathrm{mg} \mathrm{dm}{ }^{-3} ; \mathrm{H}+\mathrm{Al}=4,47 \mathrm{cmol}_{\mathrm{c}} \mathrm{dm}^{-3}$. Valores médios aos 300 dias de $\mathrm{P}=150 \mathrm{mg} \mathrm{dm}^{-3}, \mathrm{~K}=70 \mathrm{mg} \mathrm{dm}^{-3} ; \mathrm{H}+\mathrm{Al}=6,64 \mathrm{cmol}_{\mathrm{c}} \mathrm{dm}^{-3}$.

\subsection{Respostas nas Culturas}

\subsubsection{Soja}

Houve efeito significativo das relações Ca:Mg para a altura das plantas, inserção da $1^{\mathrm{a}}$ vagem, massa seca de raiz, massa seca da parte aérea, relação parte aérea:raiz, vagem com 2 grãos, vagem por planta, massa de mil grão, produção por planta, concentração e acúmulo de Ca e Mg na parte aérea da planta (Tabelas 5 e 6). As plantas mais altas, cerca de $5 \mathrm{~cm}$, cresceram em solo com relação Ca: $\mathrm{Mg}$ 05:01 e 06:01, ou seja, na presença de maiores teores de $\mathrm{Ca}$. A inserção da $1^{\mathrm{a}}$ vagem seguiu mesma tendência.

A massa seca de raiz foi superior no tratamento 04:01 seguido do tratamento 06:01, sendo estes significativamente superior aos tratamentos 01:01 e 02:01, em que a disponibilidade no solo de Ca foi menor em relação ao $\mathrm{Mg}$. A massa seca da parte aérea e por consequência a maior relação parte aérea/raiz estabeleceu-se no tratamento 05:01, em que a planta acumulou 47,28 g entre ramos e folhas, valor este que tem relação direta com a altura da planta, como já apontado. A maior constância de vagens com 2 grãos foi observada no tratamento 01:01, o que resultou também ao final em plantas com mais vagens neste tratamento, total de 51,2 vagens por planta, seguido pelo tratamento 05:01. A maior massa de 1000 grãos foi observada no tratamento 02:01 (85,9 g), seguido do tratamento 05:01 (83,6 g) e a menor massa no tratamento 01:01 (77,7 g) demostrando que a relação Ca: $\mathrm{Mg}$ do solo direta ou indiretamente interfere neste componente de produção.

A produção final de grãos foi significativamente superior em três tratamentos $(02: 01,05: 01$ e $01 ; 01)$. No tratamento 02:01, foi de 9,57 g de grãos de soja por planta, influenciado pela maior massa de mil grãos $(85,9 \mathrm{~g})$, na relação 05:01, a produção foi de $9,49 \mathrm{~g}$, resultante da maior massa de mil grãos $(83,6$ g) e da maior quantidade de vagens na planta (49 vagens) e na relação 01:01, que produziu 9,19 g, a maior quantidade de vagens por planta se destacou, com vagens de dois grãos.

As concentrações de $\mathrm{Ca}$ na planta foram superiores nos tratamentos em que o $\mathrm{Ca}$ era mais abundante no solo (tratamentos 05:01, 04:01, 06:01), o que também resultou em maior acúmulo de $\mathrm{Ca}$ na planta, respeitando esta mesma ordem em relação aos valores observados. As concentrações de $\mathrm{Mg}$ seguiram também a tendência dos tratamentos, em que os maiores valores de $\mathrm{Mg}$ no solo, nas menores relações Ca: $\mathrm{Mg}$ resultaram em maiores concentrações de $\mathrm{Mg}$ nas plantas e maior acúmulo (tratamentos 02:01 e 01:01).

\subsubsection{Milho}

Houve efeito significativo das relações Ca: $\mathrm{Mg}$ no solo na planta de milho para o diâmetro do colmo, grãos por espiga, diâmetro da espiga, massa seca de raiz, massa seca da parte aérea, relação parte aérea:raiz, massa de mil grão, produção por planta (Tabela 7).

O menor diâmetro de colmo foi observado na relação Ca: $\mathrm{Mg}$ 01:01 e os maiores valores para esta variável nos tratamentos 04:01 e 06:01, sob maior presença de Ca no solo. Espigas com menor diâmetro foram observadas na relação 06:01. A massa seca do sistema radicular foi superior nas relações Ca:Mg superiores (06:01, 04:01 e 05:01) (Tabela 7), resultado do fornecimento de $\mathrm{Ca}$ às raízes e com comportamento similar ao obtido na cultura da soja (Tabela 4). Também o acúmulo de massa na parte aérea foi superior 
nos tratamentos 04:01 e 05:01 e o menor acúmulo de massa na relação 01:01. Assim as maiores relações parte aérea:raiz estabeleceram-se nos tratamentos intermediários, decorrentes dos acúmulos nestas duas estruturas.

Tabela 5. Valores médios da altura de plantas (Alt), altura da inserção da $1^{\mathrm{a}}$ vagem $\left(1^{\mathrm{a}} \mathrm{vg}\right)$, diâmetro do caule (Diamt), grãos por planta $(\mathrm{Gr} / \mathrm{pta})$, massa seca da raiz (Mr), massa seca da parte aérea (Mpa), relação parte aérea:raiz (Pa:ra), vagens com 1, 2 e 3 grãos (Vg1, Vg2, $\mathrm{Vg} 3)$, vagens por planta (Vg/pta), massa de mil grãos (M1000) e produção de grãos por planta (Prod) em função da aplicação de calcário para calibrar as relações Ca:Mg no solo.

Table 5. Average values of plant height (Alt), height of insertion of the 1st pod ( $\left.1^{\mathrm{a}} \mathrm{vg}\right)$, stem diameter (Diamt), grains per plant (Gr / pta), root dry mass (Mr), dry mass of the aerial part (Mpa), aerial part:root ratio (Pa:ra), pods with 1, 2 and 3 grains (Vg1, Vg2, Vg3), pods per plant (Vg/pta), mass of a thousand grains (M1000) and production of grains per plant (Prod) depending on the application of lime to calibrate the $\mathrm{Ca}: \mathrm{Mg}$ ratios in the soil.

\begin{tabular}{|c|c|c|c|c|c|c|c|c|c|c|c|c|c|}
\hline TRAT & Alt & $1^{a} \mathrm{vg}$ & Diamt & $\mathrm{Gr} / \mathrm{pta}$ & $\mathrm{Mr}$ & Mpa & Pa:ra & Vg1 & $\mathrm{Vg} 2$ & Vg3 & $\mathrm{Vg} / \mathrm{pta}$ & M1000 & Prod \\
\hline (Ca:Mg) & \multicolumn{2}{|c|}{$\mathrm{cm}$} & $\mathrm{mm}$ & \multicolumn{4}{|c|}{ g } & & & & & \multicolumn{2}{|l|}{$\mathrm{g}$} \\
\hline 01:01 & $54,82 \mathrm{~b}$ & $4,82 \mathrm{~b}$ & $0,48 \mathrm{a}$ & $102,8 \mathrm{a}$ & $11,49 \mathrm{c}$ & $45,52 \mathrm{~b}$ & $3,98 \mathrm{a}$ & $10,1 \mathrm{a}$ & $30,7 \mathrm{a}$ & $10,5 \mathrm{a}$ & $51,2 \mathrm{a}$ & $77,7 \mathrm{c}$ & $9,19 \mathrm{a}$ \\
\hline 02:01 & $54,80 \mathrm{~b}$ & $5,54 \mathrm{~b}$ & $0,44 \mathrm{a}$ & $96,9 \mathrm{a}$ & $11,33 \mathrm{c}$ & $45,33 \mathrm{~b}$ & $4,00 \mathrm{a}$ & $8,3 \mathrm{a}$ & $27,5 \mathrm{~b}$ & $11,2 \mathrm{a}$ & $47,0 \mathrm{~b}$ & 85,9 a & $9,57 \mathrm{a}$ \\
\hline 03:01 & $55,40 \mathrm{~b}$ & $6,70 \mathrm{a}$ & $0,46 \mathrm{a}$ & 94,5 a & $12,75 \mathrm{~b}$ & $44,52 \mathrm{~b}$ & $3,50 \mathrm{~b}$ & $7,5 \mathrm{a}$ & $26,3 \mathrm{~b}$ & $11,7 \mathrm{a}$ & $45,4 \mathrm{~b}$ & $82,2 \mathrm{~b}$ & $8,96 \mathrm{~b}$ \\
\hline 04:01 & $53,52 \mathrm{~b}$ & $7,30 \mathrm{a}$ & $0,35 \mathrm{a}$ & $93,8 \mathrm{a}$ & $13,77 \mathrm{a}$ & $44,80 \mathrm{~b}$ & $3,26 \mathrm{~b}$ & $7,8 \mathrm{a}$ & $27,0 \mathrm{~b}$ & $10,7 \mathrm{a}$ & $45,6 \mathrm{~b}$ & $76,7 \mathrm{c}$ & $8,29 \mathrm{c}$ \\
\hline 05:01 & $59,25 \mathrm{a}$ & $6,92 \mathrm{a}$ & $0,45 \mathrm{a}$ & $98,9 \mathrm{a}$ & $11,52 \mathrm{c}$ & $47,28 \mathrm{a}$ & $4,12 \mathrm{a}$ & $10,3 \mathrm{a}$ & $27,2 \mathrm{~b}$ & $11,3 \mathrm{a}$ & $49,0 \mathrm{a}$ & $83,6 \mathrm{~b}$ & $9,49 \mathrm{a}$ \\
\hline 06:01 & $58,17 \mathrm{a}$ & $7,44 \mathrm{a}$ & $0,49 \mathrm{a}$ & $95,0 \mathrm{a}$ & $12,91 \mathrm{~b}$ & $44,94 \mathrm{~b}$ & $3,49 \mathrm{~b}$ & $9,1 \mathrm{a}$ & $25,73 \mathrm{~b}$ & $11,3 \mathrm{a}$ & $46,2 \mathrm{~b}$ & $81,1 \mathrm{~b}$ & $8,81 \mathrm{~b}$ \\
\hline CV & 2,92 & 12,95 & 10,28 & 6,65 & 4,94 & 1,70 & 5,10 & 23,49 & 6,83 & 11,85 & 7,13 & 2,30 & 6,32 \\
\hline
\end{tabular}

Médias seguidas da mesma letra na coluna não diferem estatisticamente entre si a 5\% pelo teste de Scott Knott.

Tabela 6. Valores médios das concentrações de cálcio (Ca) e magnésio $(\mathrm{Mg})$ na planta inteira e acúmulo em função da aplicação de calcário para calibrar as relações Ca:Mg no solo.

Table 6. Average values of calcium (Ca) and magnesium $(\mathrm{Mg})$ concentrations in the entire plant and accumulation as a function of the application of lime to calibrate the $\mathrm{Ca}: \mathrm{Mg}$ ratios in the soil.

\begin{tabular}{ccccc}
\hline TRAT & \multicolumn{1}{c}{$\mathrm{Ca}$} & \multicolumn{1}{c}{$\mathrm{Mg}$} & \multicolumn{1}{c}{ Ca na planta } & Mg na planta \\
\cline { 2 - 5 }$(\mathrm{Ca}: \mathrm{Mg})$ & \multicolumn{2}{c}{$\mathrm{g} \mathrm{kg}^{-1}$} & \multicolumn{2}{c}{$\mathrm{g} \mathrm{planta}^{-1}$} \\
\cline { 2 - 5 } $01: 01$ & $7,05 \mathrm{c}$ & $6,45 \mathrm{~b}$ & $0,320 \mathrm{~d}$ & $0,296 \mathrm{~b}$ \\
$02: 01$ & $12,04 \mathrm{~b}$ & $8,17 \mathrm{a}$ & $0,544 \mathrm{c}$ & $0,370 \mathrm{a}$ \\
$03: 01$ & $12,14 \mathrm{~b}$ & $6,40 \mathrm{~b}$ & $0,542 \mathrm{c}$ & $0,286 \mathrm{~b}$ \\
$04: 01$ & $14,17 \mathrm{a}$ & $6,16 \mathrm{~b}$ & $0,634 \mathrm{~b}$ & $0,278 \mathrm{~b}$ \\
$05: 01$ & $15,20 \mathrm{a}$ & $5,69 \mathrm{~b}$ & $0,720 \mathrm{a}$ & $0,270 \mathrm{~b}$ \\
$06: 01$ & $14,04 \mathrm{a}$ & $5,45 \mathrm{~b}$ & $0,628 \mathrm{~b}$ & $0,246 \mathrm{~b}$ \\
\hline $\mathrm{CV}$ & 9,83 & 11,61 & 9,61 & 12,12 \\
\hline
\end{tabular}

Médias seguidas da mesma letra na coluna não diferem estatisticamente entre si a 5\% pelo teste de Scott Knott.
Plantas que apresentaram espigas com maior quantidade de grãos (mais de 383 grãos) cresceram em solo onde as relações Ca:Mg eram maiores (tratamento 05:01 e 04:01), ao passo que na relação 01:01 as espigas tinham apenas 307 grãos. A maior massa de grãos foi observada no tratamento 01:01, porém estatisticamente igual até a relação 04:01. A menor massa dos grãos foi observada na relação mais alta de $\mathrm{Ca}: \mathrm{Mg}$.

A maior produção ocorreu no tratamento 04:01 (27,33 g), seguido do tratamento 03:01 (25,83 g), mostrando que relações próximas a 03:01 - 04:01 favorecem a produção de grãos da cultura. Esta relação foi também favorável ao acúmulo de folhas e colmo, que são os órgãos de produção e armazenamento na planta.

Tabela 7. Valores médios da altura de plantas (Alt), número de folhas (Folh), diâmetro do colmo (Diamt.C), diâmetro da espiga (Diamt.E), grãos por fileira (Gr/fil), grãos por espiga (Gr/esp), massa seca da raiz (Mr), massa seca da parte aérea (Mpa), relação parte aérea:raiz (Pa:ra), massa de mil grãos (M1000) e produção de grãos por planta (Prod) de milho em função da aplicação de calcário para calibrar as relações Ca:Mg no solo.

Table 7. Average values of plant height (Alt), number of leaves (Folh), stem diameter (Diamt.C), ear diameter (Diamt.E), grains per row (Gr/fil), grains per ear (Gr/ sp), dry mass of the root (Mr), dry mass of the aerial part (Mpa), aerial part:root ratio (Pa:ra), mass of a thousand grains (M1000) and grain production per plant (Prod) of corn in function of limestone application to calibrate the Ca:Mg ratios in the soil.

\begin{tabular}{|c|c|c|c|c|c|c|c|c|c|c|c|}
\hline \multirow{2}{*}{$\mathrm{Ca}: \mathrm{Mg}$} & Alt & Folh & Diam.C & Diam.E & $\mathrm{Gr} / \mathrm{fil}$ & Gr/esp & $\mathrm{Mr}$ & Mpa & Pa:ra & M1000 & Prod \\
\hline & $\mathrm{m}$ & & $\mathrm{mm}$ & $\mathrm{cm}$ & & \multicolumn{4}{|c|}{-------- g -------- } & \multicolumn{2}{|c|}{ 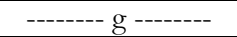 } \\
\hline 01:01 & $1,63 \mathrm{a}$ & $12,4 \mathrm{a}$ & $8,55 \mathrm{~b}$ & $3,76 \mathrm{a}$ & $21,3 \mathrm{a}$ & $307 \mathrm{~b}$ & $8,79 \mathrm{~b}$ & $28,9 \mathrm{c}$ & $3,3 \mathrm{~b}$ & $160 \mathrm{a}$ & $24,50 \mathrm{a}$ \\
\hline 02:01 & $1,69 \mathrm{a}$ & $12,6 \mathrm{a}$ & $9,60 \mathrm{a}$ & $3,74 \mathrm{a}$ & $22,1 \mathrm{a}$ & $327 \mathrm{~b}$ & $10,58 \mathrm{~b}$ & $39,9 \mathrm{~b}$ & $3,8 \mathrm{~b}$ & $152 \mathrm{a}$ & $24,68 \mathrm{a}$ \\
\hline 03:01 & $1,63 \mathrm{a}$ & $12,9 \mathrm{a}$ & $9,30 \mathrm{a}$ & $3,84 \mathrm{a}$ & $21,4 \mathrm{a}$ & $331 \mathrm{~b}$ & $9,97 \mathrm{~b}$ & $43,5 \mathrm{~b}$ & $4,4 \mathrm{a}$ & $154 \mathrm{a}$ & $25,83 \mathrm{a}$ \\
\hline 04:01 & $1,70 \mathrm{a}$ & $12,7 \mathrm{a}$ & $10,05 \mathrm{a}$ & $3,76 \mathrm{a}$ & $24,0 \mathrm{a}$ & $383 \mathrm{a}$ & $11,84 \mathrm{a}$ & $53,9 \mathrm{a}$ & $4,5 \mathrm{a}$ & $143 \mathrm{a}$ & $27,33 \mathrm{a}$ \\
\hline 05:01 & $1,66 \mathrm{a}$ & $12,4 \mathrm{a}$ & $8,80 \mathrm{~b}$ & $3,81 \mathrm{a}$ & $24,0 \mathrm{a}$ & $391 \mathrm{a}$ & $11,40 \mathrm{a}$ & $47,0 \mathrm{~b}$ & $4,2 \mathrm{a}$ & $131 \mathrm{~b}$ & $25,67 \mathrm{a}$ \\
\hline 06:01 & $1,71 \mathrm{a}$ & $12,8 \mathrm{a}$ & $9,75 \mathrm{a}$ & $3,44 \mathrm{~b}$ & $21,0 \mathrm{a}$ & $330 \mathrm{~b}$ & $12,29 \mathrm{a}$ & $41,3 \mathrm{~b}$ & $3,4 \mathrm{~b}$ & $115 \mathrm{~b}$ & $17,50 \mathrm{~b}$ \\
\hline $\mathrm{CV}$ & 3,27 & 3,92 & 8,6 & 4,06 & 10,69 & 14,88 & 10,31 & 15,85 & 15,44 & 9,83 & 11,44 \\
\hline
\end{tabular}

Médias seguidas da mesma letra na coluna não diferem estatisticamente entre si a 5\% pelo teste de Scott Knott.

\section{DISCUSSÃO}

Os valores de $\mathrm{pH}$ do solo variaram entre os tratamentos, o que ocorreu aparentemente ao acaso, pois tanto na avaliação aos 90 e 300 dias, os valores maiores observados foram nos tratamentos extremos (01:01, 05:01 e 06:01), não condizendo com um provável efeito de um calcário em particular ou da relação Ca: $\mathrm{Mg}$ buscada, já que a dose foi única em todos os vasos. Holzschuh (2007) verificou na maioria situações que maiores $\mathrm{pH}$ no solo ocorreram para a aplicação de altas doses de calcário calcítico. Clark et al.
(1997) observaram maior $\mathrm{pH}$ na relação 02:01, comparada a relações 10:01 e 20:01.

Com a observação ao longo do tempo, verifica-se que o solo apresentava $\mathrm{pH}$ inicial de 4,37 no momento da coleta na mata e, após o período de 90 dias da incubação com a dose de 8 ton ha ${ }^{-1}$ passou a apresentar em média 5,4, passando ainda para 5,7 ao final do cultivo de soja, aos 200 dias, com a maior reação do calcário. Aos 300 dias o pH médio caiu para 4,25 , apresentando maior uniformidade entre os tratamentos empregados e maior acidificação no sistema ao final. Isto se 
comprova pelos valores médios de $\mathrm{H}+\mathrm{Al}$ aos 200 dias, que foram em média de $4,7 \mathrm{cmol}_{\mathrm{c}} \mathrm{dm}^{-3}$ passando para $6,6 \mathrm{cmol}_{\mathrm{c}}$ $\mathrm{dm}^{-3}$ aos 300 dias (Tabela 4), decorrentes da aplicação de uma maior dose de $\mathrm{N}$ no milho e ao período de repouso do solo, o que deve ter provocado maior acidificação do solo.

A aplicação de fertilizantes nitrogenados causa acidificação no solo (CANTARELLA; MONTEZANO, 2010), além do balanço eletroquímico e da absorção/extração e exportação de nutrientes que as culturas necessitam, absorvendo cátions como $\mathrm{K}^{+}, \mathrm{Ca}^{2+}$ e $\mathrm{Mg}^{2+}$ e exsudando $\mathrm{H}^{+}$ na rizosfera (LANGE et al., 2019).

Os valores de $\mathrm{V} \%$ do solo não passaram de $50 \%$ na média dos tratamentos, decaindo na última avaliação para 44\%, valor menor que o proposto pelo método (67\%), evidenciando que os valores pretendidos não têm sido atingidos, como apontado por Natale et al. (2007) e Morelli et al. (1992).

Os teores de $\mathrm{Ca}, \mathrm{Mg}$ e as relações $\mathrm{Ca}: \mathrm{Mg}$ no solo aos 45, 90, 200 e 300 dias seguiram a linearidade buscada no trabalho, conforme os tratamentos propostos corroborando com Silva (1980), Moreira et al. (1999) e Medeiros et al. (2008), confirmando que se pode estabelecer os cálculos para as relações desejadas, conhecer os teores de $\mathrm{Ca}$ e $\mathrm{Mg}$ nos corretivos a serem usados e as mesmas acontecem no solo.

Aos 300 dias as relações Ca: $\mathrm{Mg}$ começaram a se estreitar, com um aumento da disponibilidade de $\mathrm{Mg}$ no solo em relação a $\mathrm{Ca}$, como pode ser visto comparando os valores absolutos de $\mathrm{Mg}$ da amostragem aos 200 dias versus 300 dias, com maiores valores para a última coleta, além dos maiores valores da \% de $\mathrm{Mg}$ na CTC. Isto é resultado do consumo maior de $\mathrm{Ca}$ nos tratamentos em que havia mais cálcio disponível (Tabela 5) e também devido a dureza das rochas. A maior dureza da dolomita (3,5 a 4,0) na escala de Mohs em relação a dureza da calcita $(3,0)$, orienta ao calcário calcítico uma reação mais rápida e maior liberação de Ca inicialmente com comportamento contrário no calcário dolomítico, que irá aumentar a liberação de $\mathrm{Mg}$ ao longo do tempo. As variações nas \% de Ca e de $\mathrm{Mg}$ na CTC também seguiram a tendência já apresentada aos 90 dias, com a maior $\%$ de Ca no tratamento 06:01, e a maior \% de $\mathrm{Mg}$ no tratamento 01:01. Segundo Yadare; Girdhar (1981), há competição entre o Ca e Mg pelos sítios de adsorção no solo, onde o Ca apresenta maior preferência em relação ao $\mathrm{Mg}$ no complexo de troca do solo, assim com o passar do tempo e a maior liberação de $\mathrm{Mg}$ decorrente da reação do calcário dolomítico, aumentará o teor de $\mathrm{Mg}$ no solo, ficando o Ca no complexo de troca. Moreira et al. (1999), estudando relações Ca:Mg no solo também apontam redução na disponibilidade de $\mathrm{Ca}$ ao longo do tempo de cultivo, decorrente do consumo de Ca pelas plantas de alfafa, que extraem alta quantidade de Ca.

As relações $\mathrm{Ca}: \mathrm{Mg}$ no solo determinaram o comportamento da cultura da soja em algumas situações, nos aspectos vegetativos com plantas mais bem desenvolvidas em altura, inserção de vagens mais altas, massa do sistema radicular e da parte aérea maiores nas relações Ca: $\mathrm{Mg} \geq 04: 01$ e, por consequência, presença de $\mathrm{Ca} \geq 4,0 \mathrm{cmol}_{\mathrm{c}} \mathrm{dm}^{-3}$, conforme mostram os resultados da amostragem aos 90 dias da incubação, data anterior ao cultivo da soja. A alta disponibilidade de Ca comprova a importância do elemento para o desenvolvimento da planta, auxiliando o crescimento do sistema radicular e no estabelecimento da cultura, pois este nutriente faz parte da parede celular, das estruturas de crescimento da planta, como ligante na calmodulina, proteína importante na regulação da atividade de muitas enzimas, e sua falta causa a atrofia do sistema radicular, inadequado desenvolvimento da gema apical (MALAVOLTA et al., 1997).

Gallo et al. (1956) verificaram acréscimo de hastes e galhos na presença de calcário dolomítico em relação a calcítico. Moreira et al. (1999) ao cultivarem alfafa sob diferentes relações $\mathrm{Ca}: \mathrm{Mg}$ não verificaram ganho de matéria seca da parte aérea após seis cortes, já a peso fresco dos nódulos e a massa seca foram significativamente superior no tratamento em que a relação $\mathrm{Ca}: \mathrm{Mg}$ no solo era de 04:01, sendo a maior relação testada pelos autores.

O número de grãos por planta, quantidade de vagens por planta e número de vagens com dois grãos foram superiores no tratamento 01:01, o que resultou numa produção de grãos estatisticamente superior neste tratamento (01:01), seguido dos tratamentos 02:01 e 05:01. Holzschuh (2007) variando proporções de calcários em três localidades não verificou diferença para produtividade da soja ao variar as relações $\mathrm{Ca}: \mathrm{Mg}$ tanto com calagem superficial ou incorporada.

Este resultado adverso ao obtido neste trabalho pode estar relacionado a possíveis stress hídricos que no presente trabalho não houveram e que podem ocorreu à campo.

Porém em campo é fundamental que a planta invista em sistema radicular mais abundante (tratamento 04:01, 06:01 e 03:01), como visto, visando tolerar condições adversas e mesmo assim produzir no final do ciclo, o que aconteceu no tratamento 05:01.

As variações nos teores de $\mathrm{Ca}$ e $\mathrm{Mg}$ foliares seguiram a tendência dos nutrientes no solo, o que corrobora com Salvador et al. (2011) estudando variações Ca: $\mathrm{Mg}$ no solo e efeitos na soja, Munoz Hernandez; Silveira (1998) ao avaliarem Ca:Mg no milho e Gomes et al. (2002) e Moreira et al. (1999) no tecido foliar das plantas de alfafa.

Plantas de milho com maior diâmetro de colmo, maior acúmulo de massa radicular e da parte aérea e mais grãos por espiga cresceram sob condições de Ca: $\mathrm{Mg}$ no solo $\geq 04: 01$, em teores de $\mathrm{Ca}$ no solo elevados, com comportamento similar ao obtido em alguns parâmetros na soja. Munoz Hernandez; Silveira (1998) trabalharam com quatro relações Ca:Mg no solo e duas saturações por bases (50 e 70\%) e observaram diferença no acúmulo de massa seca da parte aérea do milho aos 42 dias, favorável às relações 02:01 e 03:01 apenas no $\mathrm{V} \%$ de 50 , sem efeito ao se aumentar o $\mathrm{V} \%$ para 70. Clark et al. (1997) verificaram maior massa radicular no milho para relações Ca:Mg iguais a 20:10 e 10:01, comparadas as menores relações. Silva (1980) obteve maior acúmulo de massa nas raízes e na parte aérea na relação 03:01, comparada a 01:01 em milho, em dois solos distintos.

Tanto na cultura da soja como no milho, houve interferência da relação Ca: $\mathrm{Mg}$ no solo sobre a massa de grãos das culturas. Na soja, uma possível menor absorção de K pode ter ocorrido em altos teores de $\mathrm{Mg}$ no solo (01:01), como em altos teores de $\mathrm{Ca}$ (06:01), justificando a inibição competitiva entre $\mathrm{K}$, Ca e $\mathrm{Mg}$. Este comportamento foi relatado por Medeiros et al. (2008) no milho, por Salvador et al. (2011), na soja.

No milho esta condição fica ainda mais evidente, com redução aparentemente linear da massa dos grãos com o aumento do teor de Ca no solo. É sabido que o nutriente que mais favorece o enchimento de grãos é o $\mathrm{K}$ e que altos teores de $\mathrm{Ca}$ e $\mathrm{Mg}$ no solo podem interferir na absorção de $\mathrm{K}$, pela competição pelo mesmo sitio de absorção.

O resultado da análise de solo após a colheita da soja mostrou que o solo apresentava apenas $32,0 \mathrm{mg} \mathrm{dm}^{-3} \mathrm{de} \mathrm{K}$ 
(Tabela 3), valor considerado baixo, o que pode favorecido a inibição competitiva na soja, já no milho, após sua colheita os teores de $\mathrm{K}$ eram próximos a $70,0 \mathrm{mg} \mathrm{dm}{ }^{-3}$, levando a crer que no caso da gramínea o efeito de inibição é mais severo, devido a sua maior exigência de $\mathrm{K}$.

Fancelli; Dourado-Neto (2004) detalham a importância do cálcio no desenvolvimento da cultura do milho e apontam como principais sintomas de deficiência a redução do crescimento radicular, clorose em folhas novas, morte de extremidade de raízes e menor fecundação sendo o elemento essencial para o crescimento e aprofundamento de raízes, vital para germinação do tubo polínico e indispensável à formação das paredes celulares na planta. Os resultados mostraram que plantas de milho cultivadas nas relações Ca:Mg mais baixas (01:01, 02:01 e 03:01), em que o Ca no solo era $\leq 3,2 \mathrm{cmol}_{\mathrm{c}} \mathrm{dm}^{-3}$ aos 200 dias (análise antes do cultivo) apresentaram menor quantidade de grãos por planta, possivelmente decorrente da menor fecundação; massa do sistema radicular menor, devido ao menor fornecimento de Ca o que resultou diretamente na menor massa da parte aérea, comportamento similar ao apontado pela literatura.

\section{CONCLUSÕES}

As relações $\mathrm{Ca}: \mathrm{Mg}$ estipuladas com as misturas dos corretivos foram atingidas e permaneceram no solo por praticamente 300 dias, mesmo após os cultivos da soja e do milho;

O acúmulo de massa seca radicular e da parte aérea da soja e do milho foi favorecido nas relações Ca:Mg iguais ou superiores a 04:01;

A soja produziu mais grãos na relação 02:01, 01:01 e 05:01 e o milho só reduziu sua produção de grãos na relação 06:01, decorrente da menor massa de mil grãos nesta relação com possível influência da menor disponibilidade de $\mathrm{K}$, devido a inibição competitiva.

\section{AGRADECIMENTOS}

A SINECAL (Sindicato das Indústrias de Extração de Calcário de Mato Grosso) pelo apoio no estudo.

\section{REFERÊNCIAS}

ALVAREZ V. V. H.; NOVAIS, R. F.; BARROS, N. F.; CANTARUTTI, R. B.; LOPES, A. S. Interpretação dos resultados das análises de solo. In: Comissão de Fertilidade do Solo do Estado de Minas Gerais CFSEMG. Recomendação para o uso de corretivos e fertilizantes em Minas Gerais: $5^{\mathbf{a}}$ aproximação. Viçosa, 1999. p. 25-32.

CANTARELLA, H.; MONTEZANO, Z. F. Nitrogênio e Enxofre. In: PROCHNOW, L. I.; CASARIN, V.; STIPP, S. R. (Orgs.). Bo as Práticas para Uso Eficiente de Fertilizantes. 1ed. Piracicaba: International Plant Nutrition Institute, 2010, v. 2, p. 1-64.

CLARK, R. B.; ZETO, S. K.; RITCHEY; K. D.; BALIGAR, V. C. Maize growth and mineral acquisition on acid soil amended with flue gas desulfurization byproducts and magnesium. Communications in Soil Science and Plant Analysis, v. 28, p. 1441-1459, 1997. DOI: https://doi.org/10.1080/00103629709369886

DUARTE, A. P.; ABREU, M. F.; FRANCISCO, E. A. B.; GITTI, D. C.; BARTH, G.; KAPPES, C. Concentração e exportação de nutrientes nos grãos de Milho. Informações agronômicas, n. 163, p. 12-16, set. 2018.

FANCELLI, A. L.; DOURADO-NETO, D. Produção de milho. Guaíba: Agropecuária, 2004. 360 p.

FERREIRA, D. F. Sisvar: um sistema computacional de análise estatística. Ciência e Agrotecnologia, Lavras, v. 35, n. 6, p. 1039-10422011. DOI: https://doi.org/10.1590/S1413-70542011000600001.

GALLO, J. R.; CATANI, R. A.; GARGANTINI, H. Efeito de três tipos de calcários na reação do solo e no desenvolvimento da soja. Bragantia, Campinas, v. 15, n. único, p. 121-130, 1956. DOI: http://dx.doi.org/10.1590/S0006-87051956000100012

GOMES, F. T.; BORGES, A. C.; NEVES, J. C. L; FONTES, P. C. R. Influência de doses de calcário com diferentes relações cálcio:magnésio na produção de matéria seca e na composição mineral da alfafa. Pesquisa Agropecuária Brasileira, Brasília, v. 37, n. 12, p. 17791786, 2002. DOI: http://dx.doi.org/10.1590/S0100204X2002001200015

HOLZSCHUH M. J. Eficiência de calcário calcítico e dolomítico na correção da acidez de solos sob plantio direto. 85f. 2007. Dissertação [Mestrado em Ciência do Solo) - Universidade Federal de Santa Maria, Santa Maria, 2007.

LANGE, A.; ZANDONADI, R. S.; GOBBI, F. C. Distribuição horizontal da fertilidade do solo em sistemas de tráfego controlado com fertilização em linha. Nativa, Sinop, v. 7, n. 3, p. 251-255, 2019. DOI: https://doi.org/10.31413/nativa.v7i3.7639

MALAVOLTA, E. Elementos da nutrição mineral de plantas. São Paulo: Editora Agronômica Ceres, 1980. 251p.

MALAVOLTA, E.; VITTI, G. C.; OLIVEIRA, S. A. Avaliação do estado nutricional das plantas: princípios e aplicações. 2 ed. Piracicaba: POTAFOS, 1997. 319p.

MEDEIROS, J. C.; ALBUQUERQUE, J. A.; MAFRA, A. L.; ROSA, J. D.; GATIBONI, L. C. Relação cálcio:magnésio do corretivo da acidez do solo na nutrição e no desenvolvimento inicial de plantas de milho em um Cambissolo Húmico Álico. Semina: Ciências Agrárias, Londrina, v. 29, n. 4, p. 799-806, 2008. DOI: http:/ /dx.doi.org/10.5433/1679-0359.2008v29n4p799

MOORE, D. P.; OVERSTREET, R.; JACOBSON, L. Uptake of magnesium and its interactions with calcium in excised barley roots. Plant Physiology, Rockville, v. 36, n. 3, p. 290-295, 1961.

MOREIRA, A.; CARVALHO, J. G. de; EVANGELISTA, A. R. Influência da relação cálcio:magnésio do corretivo na nodulação, produção e composição mineral da alfafa. Pesquisa Agropecuária Brasileira, Brasília, v. 34, n. 2, p. 249-255, 1999. DOI: https://doi.org/10.1590/S0100204X1999000200014

MORELLI, J. L.; DALBEN, A. E.; ALMEIDA, J. O. C.; DEMATTÊ, J. L. I. Calcário e gesso na produtividade da cana-de-açúcar e nas características químicas de um latossolo de textura média álico. Revista Brasileira de Ciência do Solo, Viçosa, v. 16, p. 187-194, 1992.

MUNOZ HERNANDEZ, R. J.; SILVEIRA, R. I. Efeitos da saturação por bases, relações ca:mg no solo e níveis de fósforo sobre a produção de material seco e nutrição mineral do milho (Zea mays L.). Scientia Agricola, 
Piracicaba, v. 55, n. 1, p. 79-85, jan. 1998. DOI: https://doi.org/10.1590/S0103-90161998000100014.

NATALE, W.; PRADO, R. M.; ROZANE, D. E.; ROMUALDO, L. M. Efeitos da calagem na fertilidade do solo e na nutrição e produtividade da goiabeira. Revista Brasileira de Ciência do Solo, Brasília, v. 31, n. 6, p. 1475-1485, 2007. DOI: https://doi.org/10.1590/S010006832007000600024

RAIJ, B. Van; CANTARELlA, H.; QUAGGIO, J. A.; FURLANI, A. M. C. (Eds). Recomendações de adubação e calagem para o Estado de São Paulo. Campinas: Instituto Agronômico, 1996. 285p.

SALVADOR, J. T.; CARVALHO, T. C.; LUCCHES, L. A. C. Relações cálcio e magnésio presentes no solo e teores foliares de macronutrientes. Revista Acadêmica Ciências Agrárias e Ambiental, Curitiba, v. 9, n. 1, p. 27-32, 2011

SEDIYAMA, T. Tecnologias de produção e usos da soja. Londrina: Macenas, 2009. 314p.

SILVA, J. E. Balanço de cálcio e magnésio e desenvolvimento do milho em solos sob Cerrado. Pesquisa Agropecuária Brasileira, Brasília, v. 15, n.3, p. 329-333, 1980.

TOME JUNIOR, J. B.. Manual para Interpretação de Análises de Solos. Guaíba: Gráfica e Editora Agropecuária, 1996. 148p.

YADARE, J. S. P.; GIRDHAR, I. K. The effects of different magnesium:calcium rations and sodium adsorption ratio values fo leaching water on the properties of calcareous versus noncalcareus soils. Soil Science, v. 131, p. 194 198, 1981. 